\title{
The Design and Control Simulation of Trans-femoral Prosthesis Based on Virtual Prototype
}

\author{
Hualong Xie ${ }^{1}$, Guocun $\mathrm{Kang}^{2}$ and Fei $\mathrm{Li}^{3}$ \\ ${ }^{1}$ School of Mechanical Engineering \& Automation, Northeastern University \\ Shenyang, Liaoning, 110819, P.R. China \\ ${ }^{2}$ School of Mechanical Engineering \& Automation, Northeastern University \\ Shenyang, Liaoning, 110819, P.R. China \\ ${ }^{3}$ School of Information Science \& Engineering, \\ Shenyang University of Technology, Shenyang, Liaoning, 110870, P.R. China \\ 1'hlxie@mail.neu.edu.cn, ${ }^{2}$ lifeisut@163.com, ${ }^{3}$ kangguocun1203@163.com
}

\begin{abstract}
Intelligent bionic leg (IBL) is an advanced trans-femoral prosthesis. It can compensate basic movement function of amputees. The prototype of IBL is introduced in the paper. The existing torque devices of knee joint are analyzed and dynamics model of IBL is deduced. An ideal test-bed of IBL is proposed and it can generate the various moments of human healthy leg. Using software Pro/E, ADAMS and MATLAB/Simulink, the virtual prototype of IBL and its simulation platform is built. The combined control simulation based on neural network indicates that IBL controlled by magneto-rheological (MR) damper can track human healthy leg's gait well.
\end{abstract}

Keywords: Trans-femoral prosthesis, Control simulation, Virtual prototype

\section{Introduction}

The world of medical devices is rapidly expanding with the aim of improving the life-style of people [1]. Due to advancements in medical technology, the number of fatalities associated with traumatic accidents or war-time injuries has been severely reduced. Injuries that once would have cost a people their lives now result in amputation [2]. The 2nd national sampling investigation of the disabled indicates that it has at least 24120 thousand people with physical disabilities in china and the lower limb amputees are about 440 thousand [3]. In the US, around 1.6 million people live with limb loss [4]. About $97 \%$ of all vascular limb loss is lower-limb amputations, of which $25.8 \%$ are above-knee $(\mathrm{A} / \mathrm{K})$ amputations.

The important way of amputees to compensate the basic movement function is dressing with lower limb prostheses, especially trans-femoral prosthesis. In both literature and market, different kinds of trans-femoral prostheses are presented and they can be classified as follows [5]:

(1) Passive, i.e., not actuated. These prostheses can be considered efficient from the mechanical point of view but the overall efficiency is hampered by the considerable amount of extra metabolic energy consumption. Moreover, due to the constant mechanical characteristics, these devices can not adapt to different conditions. 
(2) Controlled by means of internal, intrinsically passive, actuators. These prostheses use external power to adapt their dynamics to different gait pattern, such as electro-rheological or magneto-rheological damper.

(3) Active (powered), i.e., actuated - These prostheses are capable to inject power in order to provide active ankle push-off generation, so to reduce the extra metabolic energy consumption.

Intelligent bionic leg presented in the paper is an advanced trans-femoral intelligent prosthesis in the area of rehabilitation medicine [6]. It is semi-active controlled by MR damper, has good humanoid characteristics and its working style is similar to physiological mechanism of human normal leg. According to change of human walking speed and knee angle, IBL can automatically adjust control torque of knee joint. Thus the gait of IBL can match human normal gait well in symmetry. In the following, the mechanism design, dynamics modeling, simulation platform and control simulation will be presented in detail.

\section{Prosthesis Design}

The prototype of IBL developed by robotics project group at Northeastern University, China, is shown in Figure 1.

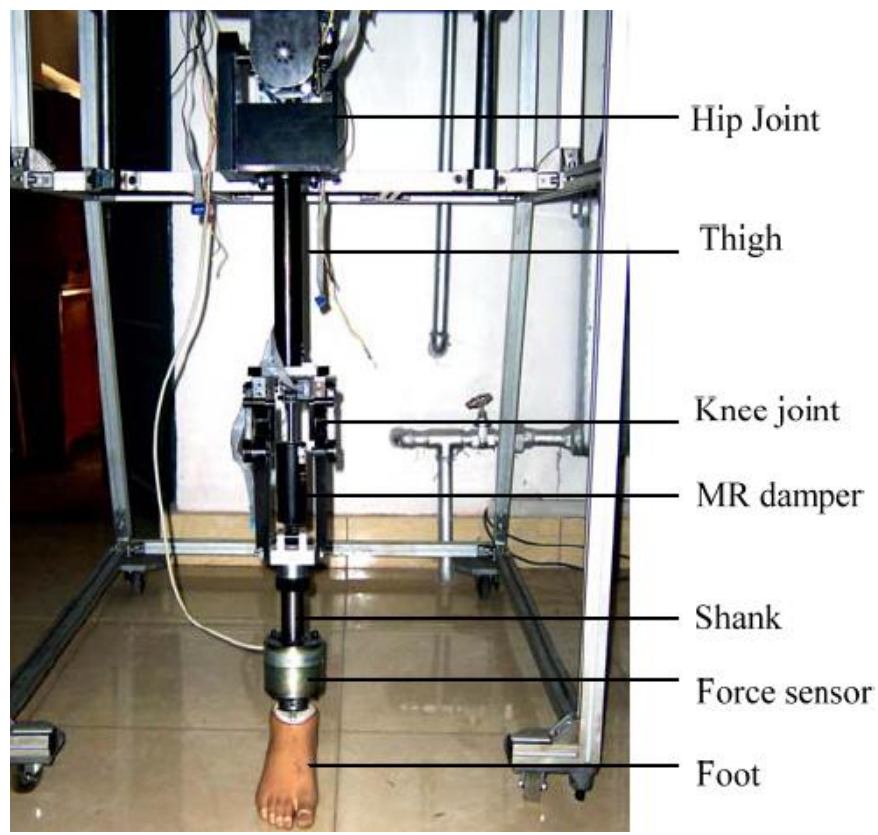

\section{Figure1. The prototype of trans-femoral intelligent prosthesis}

Its structure includes hip joint, thigh, bionic knee joint, shank and flexible prosthetic foot. Prosthetic foot is fixed to shank and ankle joint has no degree of freedom. The bionic knee joint is semi-controlled by MR damper to adjust rotation performance. MR damper can provide biggish damper force and lesser resilience to insure IBL track human natural gait well. Six-axis force sensor is used to detect information of ground reactive force. Compared to knee joint with single axis, four-bar bionic knee mechanism which is adopted in IBL has many advantages which are shown in Figure 2: 
(1) The instantaneous center of rotation (ICR) is not fixed and can change along with the change of knee joint. It can well simulate "J" curve of ICR of human normal knee.

(2) During swing phase, it can effectively shorten the length of IBL, improve the lift height of feet and avoid collisions with the ground obstacles. It matches the natural movement of human leg well.

(3) When amputees dressed with IBL are in mid-swing or sits down, the ICR of IBL knee is down to normal position and can effectively improve amputees' posture. Thus amputees can keep both legs in the same posture.

(4) It can effectively improve the stability during support phase. The four bar linkage of knee joint can make its ICR naturally after the load line by means of its mechanical structure.

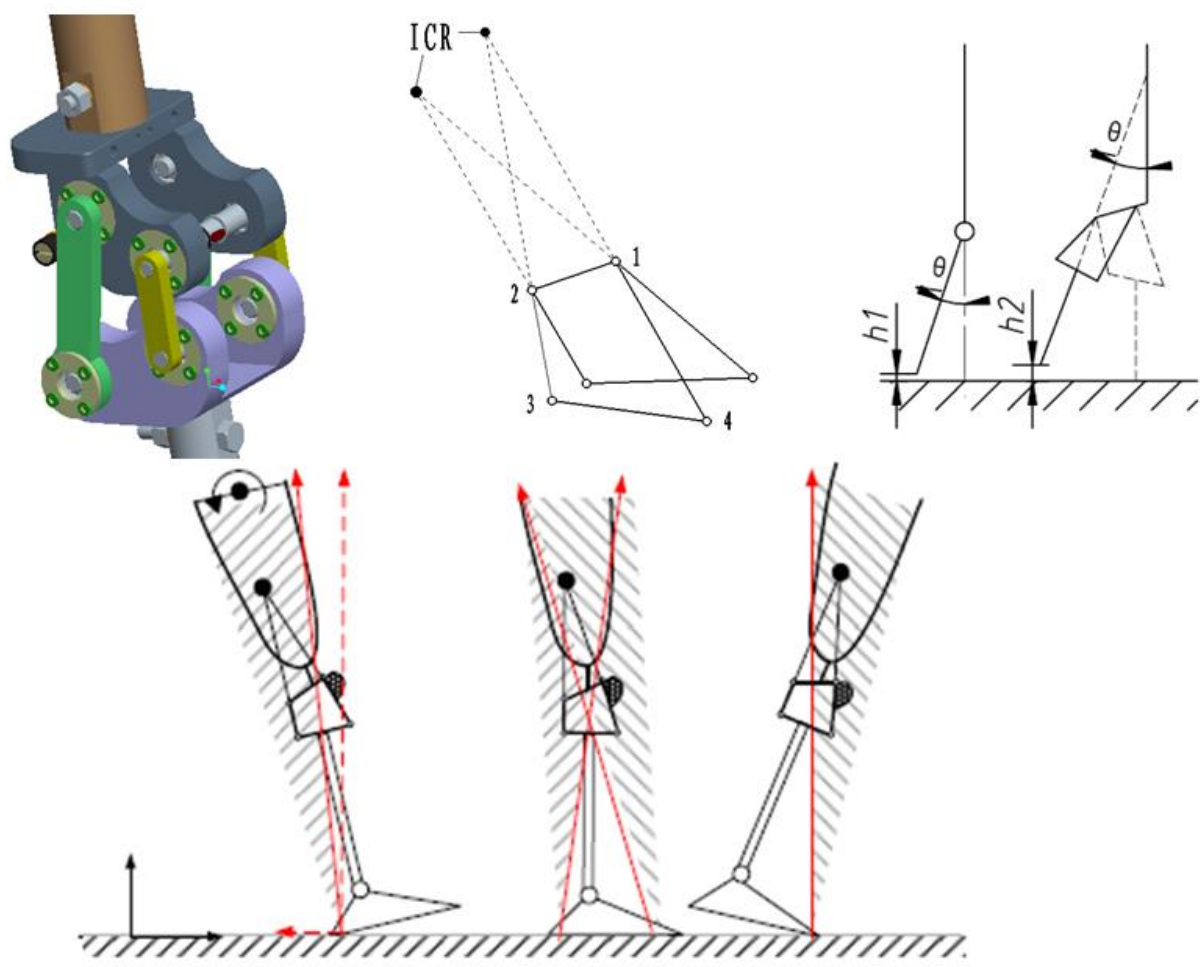

Figure 2. The virtual prototype and characteristic description of four-bar bionic knee mechanism

The torque control of knee joint is very crucial for the development of IBL. In the existing torque devices of knee joint, the constant friction is the simplest one. However, the constant torque value does not meet changes of torque requirement during whole gait cycle. Hydraulic damping device can greatly improve the torque during swing phase and the damping force can be increased when walking speed increases. But its structure is complex and machining precision is high. Pneumatic damping device can overcome the shortcoming of these two devices and is widely used. However, its volume is generally large and the available torque is limited, thus its function is limited. With the development of computer technology, microelectronics and materials science, intelligent driving devices, such as ER damper, MR damper, artificial muscle and shape memory alloy appear. MR damper is considerable potential for semi-active control and 
has a series of advantages, such as simple structure, small volume, smart response, low energy consumption and large resistance, etc. The biomedical research indicates that semi-active control of knee joint has advantages of active joint and passive joint. It matches biological mechanism of human leg well and is an ideal choice of IBL.

\section{Dynamics Modeling of IBL}

IBL model and its parameters are shown in Figure 3. $T_{2}$ is control input torque of hip joint and $T_{3}$ is control input torque of knee joint. Since flexible prosthetic foot is fixed to shank, it can be considered with shank as one part.

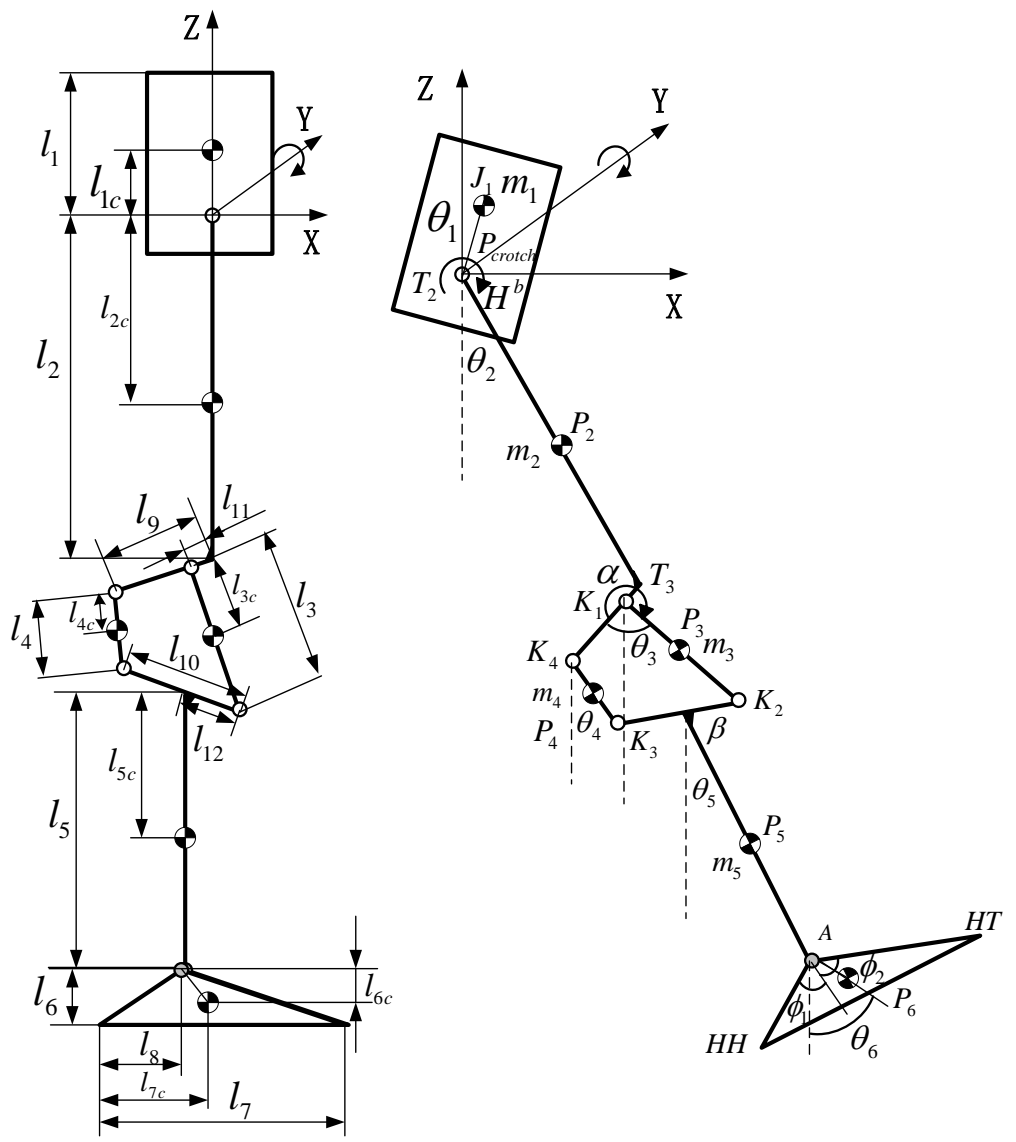

Figure 3. IBL model and its parameters

Constrain of four-bar link mechanism can be written as follows:

$$
f:-l_{9} e^{j\left(\theta_{2}-\alpha\right)}-l_{3} e^{j \theta_{3}}+l_{10} e^{j\left(\theta_{5}-\beta\right)}+l_{4} e^{j \theta_{4}}=0
$$

When trans-femoral intelligent prosthesis is in swing phase, thigh and shank swing, and hip joint follows the crotch of human moving according to certain track. The dynamics equation above can be written as follows:

$$
M_{s w}^{b}(q) \ddot{q}+C_{s w}^{b}(q) \dot{q}^{2}+G_{s w}^{b}(q)=B_{s w}^{b} T+\Phi^{T}(q) \lambda
$$


Where

$$
\begin{aligned}
& M_{S W}=\left[\begin{array}{llll}
M_{11} & M_{12} & M_{13} & M_{14} \\
M_{21} & M_{22} & M_{23} & M_{24} \\
M_{31} & M_{32} & M_{33} & M_{34} \\
M_{41} & M_{42} & M_{43} & M_{44}
\end{array}\right]: \text { inertia matrix } \\
& C_{S W}=\left[\begin{array}{llll}
C_{11} & C_{12} & C_{13} & C_{14} \\
C_{21} & C_{22} & C_{23} & C_{24} \\
C_{31} & C_{32} & C_{33} & C_{34} \\
C_{41} & C_{42} & C_{43} & C_{44}
\end{array}\right]: \text { centripetal and coriolis torques } \\
& G=\left[\begin{array}{c}
G_{1} \\
G_{2} \\
G_{3} \\
G_{4}
\end{array}\right]: \text { vector of the gravitational forces } \\
& B_{S W}=\left[\begin{array}{cccc}
1 & -1 & -1 & 0 \\
0 & 1 & 0 & -1 \\
0 & 0 & 1 & -1 \\
0 & 0 & 0 & 1
\end{array}\right]: \text { coefficient matrix of driving torques } \\
& T=\left[\begin{array}{llll}
T_{2}^{b} & T_{3}^{b} & 0 & 0
\end{array}\right]^{T}, q=\left[\begin{array}{llll}
\theta_{2}^{b} & \theta_{3}^{b} & \theta_{4}^{b} & \theta_{5}^{b}
\end{array}\right]^{T} \\
& \Phi^{T}(q) \lambda \text { is constrain torques. }
\end{aligned}
$$

\section{The Scheme Design and Virtual Prototype Modeling of IBL Simulation Platform}

To guarantee the control performance of IBL, repetitive walking test of amputees with IBL is necessary. However, IBL test will exhaust the amputees. It is not only costly but also painful to human subjects. It may even lead to accidental harm to amputees. In order to provide an idea test-bed for IBL, the research of biped robot and intelligent prosthesis is integrated and a new-style humanoid robot named biped robot with heterogeneous legs (BRHL) which is shown in Figure 4 is proposed by the robotics group at Northeastern University, China [7, 8].

BRHL consists of two legs with different functionalities. One is artificial leg which is used to simulate an amputee's healthy leg, and the other is bionic leg which is used to investigate advanced intelligent prosthesis used by amputees. The artificial leg has 6 degrees of freedom (3 DOF hip, 1 DOF knee and 2 DOF ankle). The bionic leg includes an artificial leg hip joint, IBL and flexible prosthetic foot. BRHL can simulate amputees walking with trans-femoral prosthesis and it can generate the various moments of human for the test of IBL.

Since the artificial leg of BRHL simulates human healthy leg to provide ideal natural gait for the test of IBL. Its design focuses on personification. Compared to common biped robot, 
the knee joint of BRHL artificial leg adopts four-bar bionic mechanism and pneumatic muscle actuator. Thus the design of artificial leg ensures it can simulate the biological structure and muscle-driven mode of human knee joint well. A virtual prototype of simplified BRHL and its artificial leg is shown in Figure 5 and Figure 6.

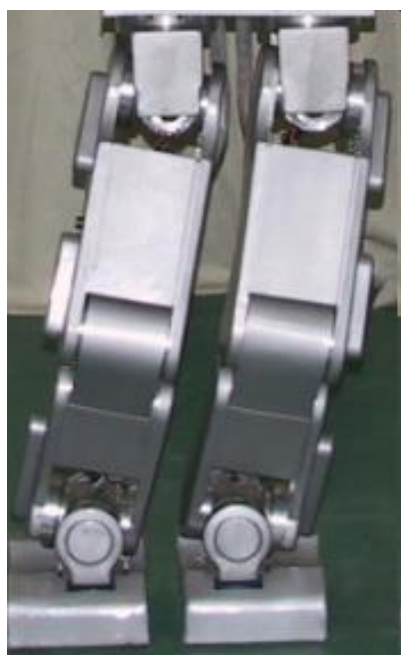

Biped robot

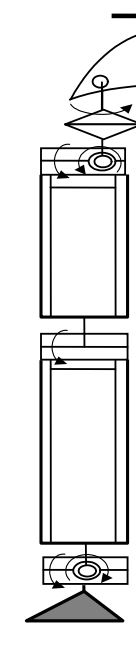

Artificial leg

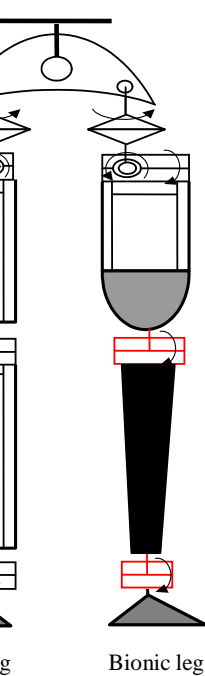

BRHL

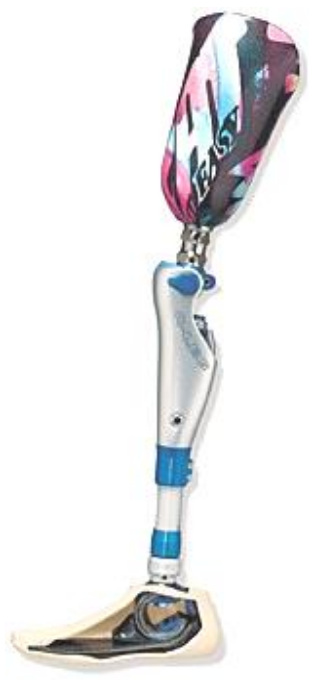

Intelligent prosthesis

Figure 4. Scheme of biped robot with heterogeneous legs

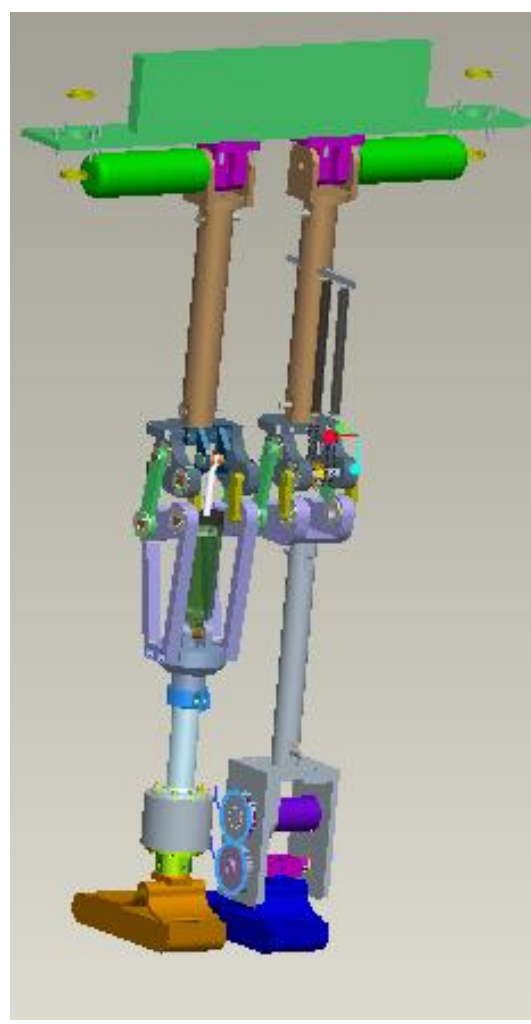

Figure 5. Virtual prototype of simplified BRHL 


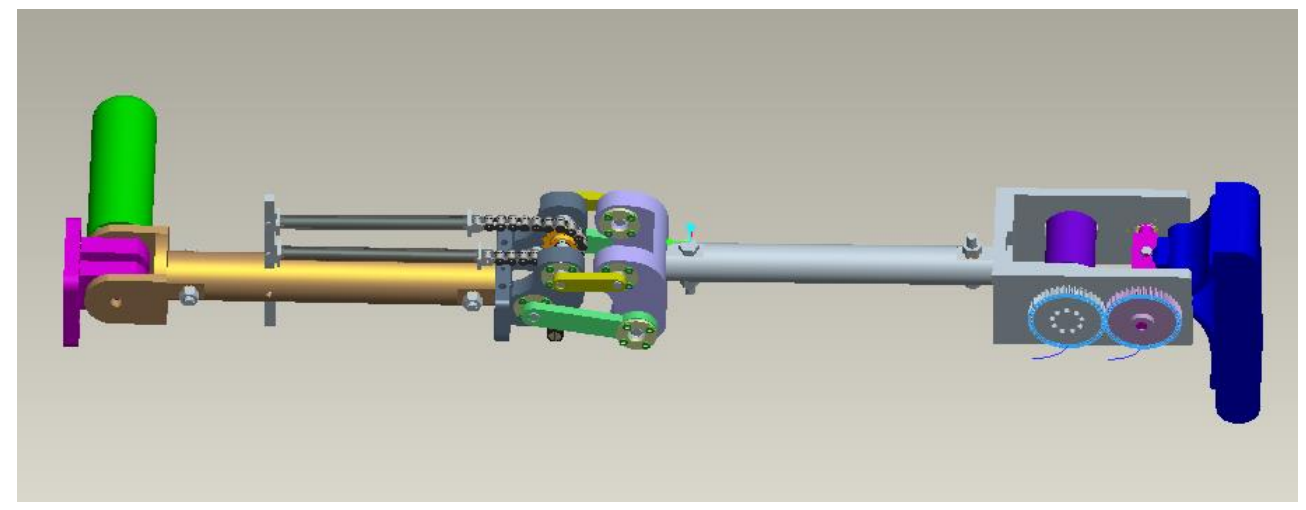

Figure 6. Virtual prototype of simplified BRHL artificial leg

\section{Virtual Prototype Modeling and Control Simulation}

When amputee dressed with IBL walks, the generation of variedly active movements and the maintenance of walking stability are done by the disabled itself. Based on BRHL, we mainly research gait tracking of IBL to human healthy leg and coordinated control of dual legs. Considering above situation, we first develop a simplified simulation platform of IBL which simulates plane walk of amputees.

The simulation platform of IBL can be divided into the mechanical system, driving device, control system, sensor system and ground environment. The information transmission of above five parts can be shown in Figure 7.

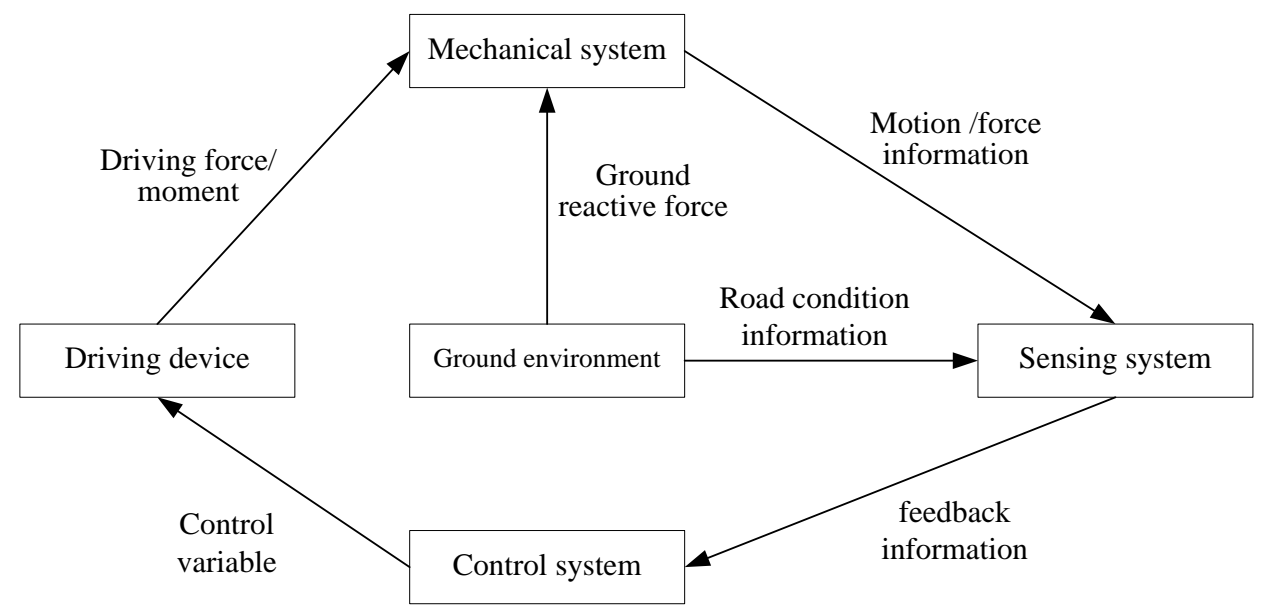

Figure 7. Information translation between IBL simulation platform

\subsection{Virtual prototype modeling}

The mechanical system of IBL and its simulation platform is more complex. Its threedimensional solid model is established in software Pro/E and is imported in software ADAMS. The geometric constraints, force constraints and joints are also added. Thus the kinematics and dynamics model can be automatically generated to build basic mechanical system model. Flexible body can be created by two methods in ADAMS. The first is using its interface module ADAMS / Flex to introduce modal neutral file created by finite element software. The second is using its ADAMS / AutoFlex module. The first 
method is suitable for stress-strain analysis of flexible body and has high accuracy, but its steps are more complicated. In this paper, flexible prosthetic foot is created by ADAMS / AutoFlex module. The virtual prototype of simplified BRHL is shown in Figure 5.

The ground environment model under various road conditions (flat, ramp, stairs, etc.) can be established in ADAMS. By establishing the contact constraints between ground and IBL foot, the relationship of geometric constraints between them can be automatically added according to there shape. The collision model and friction model between IBL foot and ground can be automatically established by contact constraints. By reasonable parameters configuration, the support and compact between foot and different roads can be simulated. The accuracy of collision model and friction model will affect the simulation accuracy. Based on superiority-inferiority analysis of different contact modeling methods, the continuous contact force method based on penalty function is adopted in the paper.

Perception system is an indispensable module of IBL. It has a variety of sensors to detect current robot posture and environmental information. Virtual perception system can get the internal and external information of IBL by measure under simulation environment. Using measure function of ADAMS, perception system of IBL can be created. It can measure angle, angular velocity, angular acceleration of any joint. Furthermore, it can measure movement and contact force of any point. Measure function of ADAMS can simulate sensors of real system and acts as a feedback (control object output) in control simulation module.

The different controller simulation module can be established in Simulink using software MATLAB for variable control algorithms. Through control interface between ADAMS and MATLAB, mechanical system of IBL and its simulation platform cab be imported into MATLAB as control object which is shown in Figure 8.

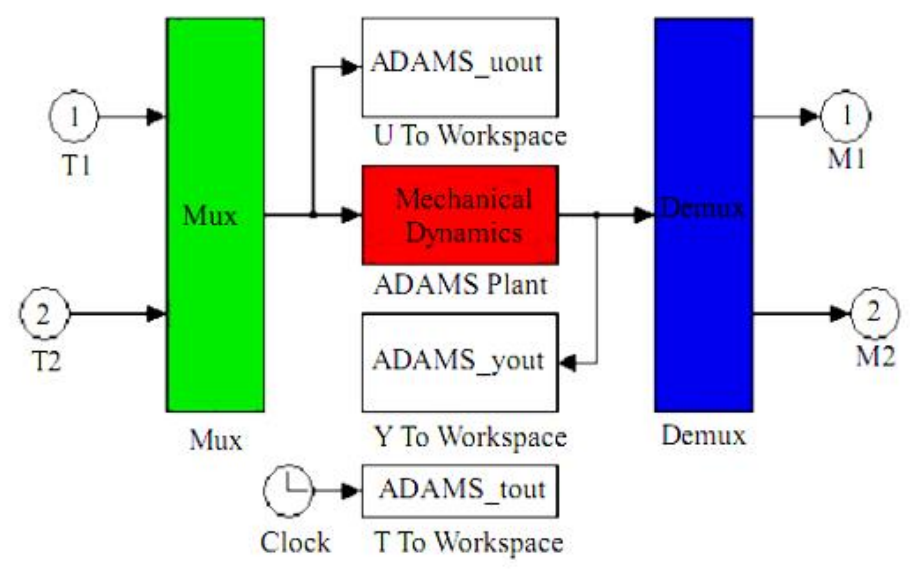

Figure 8. Simulation model of control system

\subsection{Neural Network control}

To solve arbitrary gait control of IBL, adaptive control based on system identification of neural network is proposed in this paper. Since the hardware system of IBL directly provides functions to achieve PID control and the computing is fast, convenient and accurate, PID control based on neural networks is adopted. Neural networks is used to identify system model and parameters gained is used to adjust P, I, D parameters in real-time to achieve gait tracking. Recurrent neural network has internal feedback and has strong robustness not only to the change of system parameters and external disturbance, but also to structure changes of 
system. It is well suitable for the control of complex and uncertain system. Diagonal recurrent neural network (DRNN) based on recursive prediction error (RPE) algorithm can get better accuracy than that using static BP network to complex nonlinear system modeling. So DRNN using RPE algorithm is a good choice of IBL control.

Natural gait data of artificial leg is obtained from software APAS of Ariel Dynamics Co. The angle is absolute angle. The gait tracking of IBL knee joint is shown in Figure 9. The simulation indicates that the control effect is well in a gait cycle. Though certain errors exit when joint angle dramatically changes, it can guarantee the basic movement of IBL. This control method can get good effect when generating gait does not exist in gait database.

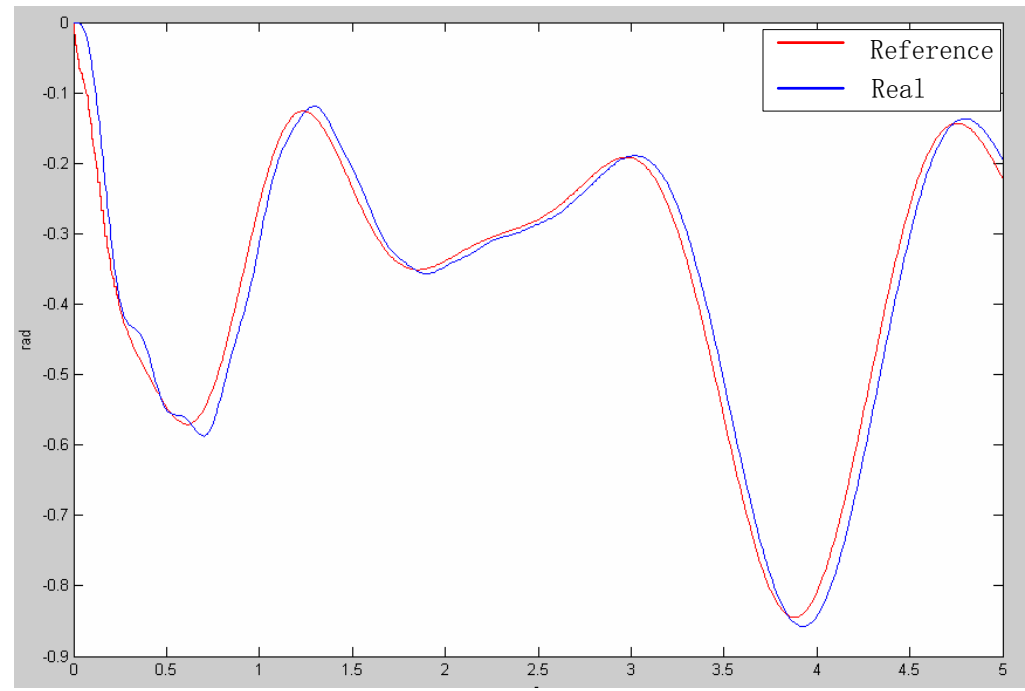

Figure 9. Gait tracking of IBL knee joint

\section{Conclusions}

Trans-femoral prosthesis has great social and economy benefit. Its research integrates advanced technologies, namely mechanism engineering, electrical engineering, sensors, signal processing, control engineering, bioengineering, materials and intelligent prosthesis etc. The structure and control model of artificial leg with four-bar bionic mechanism and pneumatic muscle actuator are similar to that of human's knee with muscle and ligament. BRHL with multi-axis knee artificial leg can generate the various moments of human healthy leg and is an ideal test-bed of IBL. Using software Pro/E, ADAMS and MATLAB/Simulink, the virtual prototype of IBL and its simulation platform can be built. The combined control simulation indicates that IBL controlled by MR damper can track human healthy leg's gait well. The control effect of prototype in practice should be further studied.

\section{Acknowledgements}

Financial supports from National Natural Science Foundation of China (ID 51105070), the Fundamental Research Funds for the Central Universities (ID N120403002) and Shenyang Science \& Technology Program Project (ID F11-264-1-55) are highly appreciated. 


\section{References}

[1] V. Luciano, E. Sardini, M. Serpelloni and G. Baronio, "Analysis of an Electromechanical Generator Implanted in a Human Total Knee Prosthesis", Proceedings of IEEE Sensors Applications Symposium, (2012) February 7-9; Brescia, Italy.

[2] M. V. Pillai, H. Kazerooni and A. Hurwich, "Design of a Semi-active Knee-ankle Prosthesis", IEEE International Conference on Robotics and Automation, (2011) May 6-13; Shanghai, China.

[3] S. Y. Gong, P. Yang, L. Song and L. L. Chen, "Simulation of Swing Phase Dynamics in Trans-femoral Prostheses Based on MATLAB", Journal of Hebei University of Technology, vol. 40, no. 2, (2011), pp. 6-9.

[4] G. K. Ziegler, E. J. MacKenzie, P. L. Ephraim, T. G. Travison and R. Brookmeyer, "Estimating the Prevalence of Limb Loss in the United States-2005 to 2050", Archives of Physical Medicine and Rehabilitation, vol. 89, no. 3, (2008), pp. 422-429.

[5] R. Unal, R. Carloni, E. E. G. Hekman, S. Stramigioli and H. F. J. M. Koopman, "Biomechanical Conceptual Design of a Passive Transfemoral Prosthesis", 32nd Annual International Conference of the IEEE EMBS, (2010) August 31 - September 4; Buenos Aires, Argentina.

[6] X. H. Xu, B. R. Wang and J. D. Tang, "A New Kind of Robot Model - Biped Robot with Heterogeneous Legs", High Technology Letter, vol. 44, no. 2, (2004), pp. 55-88.

[7] H. L. Xie, Z. Z. Liang, F. Li and L. X. Guo, "The Knee Joint Design and Control of Above-knee Intelligent Bionic Leg Based on Magneto-rheological Damper”, International Journal of Automation and Computing, vol. 7, no. 3, (2010), pp. 277-282.

[8] J. Cheng, H. Song, L. N. Hao and X. H. Xu, "Research on Co-simulation of Biped Robot with Heterogeneous Legs", Journal of System Simulation, vol. 19, no. 21, (2007), pp. 4953-4956.

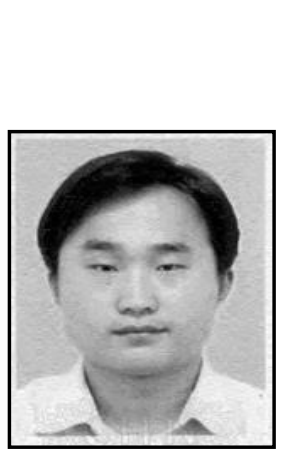

\section{Authors}

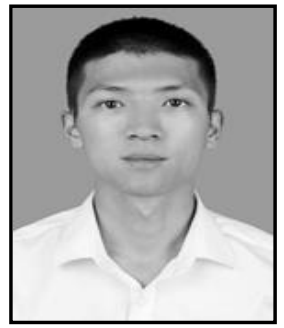

Hualong Xie. He received his bachelor degree in mechatronic Engineering, Master degree in Mechanical Design and Theory and $\mathrm{PhD}$ degree in Control Theory and Control Engineering from Northeastern University, China, in 2000, 2003 and 2006, respectively. Since 2010, he is an associate professor at Northeastern University. His research interests include robot, intelligent control, intelligent bionic leg and biomechanics.

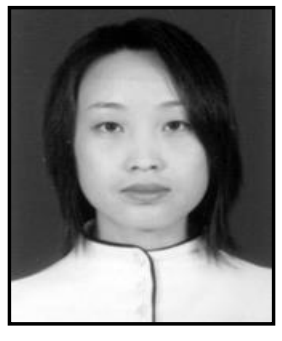

Guocun Kang. He received his bachelor degree in Mechanical Design and Theory from Hebei United University. Now he is a postgraduate at School of Mechanical Engineering \& Automation in Northeastern University, China. His current research interests include robot and intelligent control.

Fei Li. She received her bachelor degree in measurement and control technology and instrumentation, master degree in control theory and control engineering from Shenyang Institute of Chemical Technology, China, in 2001 and 2004, respectively, and the Ph.D. degree in pattern recognition and intelligent system from Northeastern University, China, in 2007. Currently, she is a lecturer in the School of Information Science \& Engineering at Shenyang University of Technology, China. Her research interest covers robotics, control theory and computer vision. 